\title{
Grid Research on Desktop Type Software for Spatial Information Processing
}

\author{
Guoqing Li, Dingsheng Liu, and Yi Sun \\ 45 BeiSanHuanXi Road, P.O. Box 2434, Beijing, 100086, China \\ Open laboratory, Remote Sensing Satellite Ground Station, China Academic of Sciences \\ \{gqli, dsliu, ysun\}@ne.rsgs.ac.cn
}

\begin{abstract}
In the research works of spatial information grid, the grided modification of desktop style processing software is very important. Based on the analysis of such software structure, paper gives its technical strategy. Function Grided modifying is the acceptable method instead of whole package grided. Within the research, PCI and PIPS are brought as two kinds examples. Some key technology is followed being discussed and an experiment is given to show an interested result.
\end{abstract}

\section{Desktop Processing Resources and PCI Software}

In spatial information processing field, workstations are the main computing platform. With them, desktop style processing is using widely. Desktop software can work in single powerful workstation with support of other peripheral equipments, such as plotter, printer and tape reader etc. Users can do their simple processing works in such machine. With the development of user's requirement, computer has to handle large sale of data, high complex arithmetic within a limited time. In addition, local data and software and computing capability are found not sufficient for use, network cooperation and resource sharing is need.

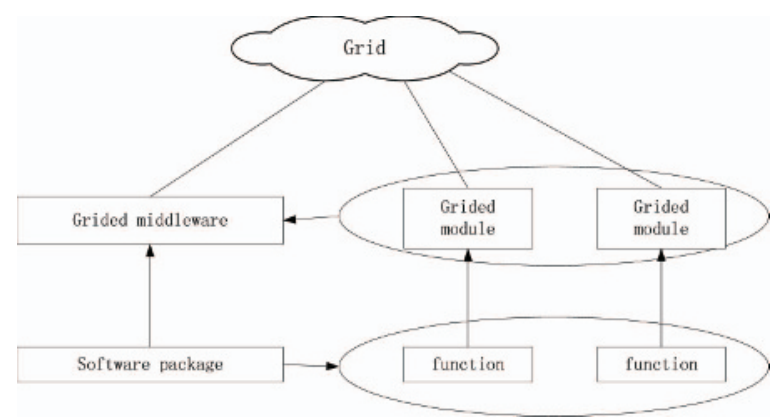

Fig. 1. How to grid a desktop software package

Grid is a method to realize such resource cooperation. With grid, users hope they can use a processing function only with their requirement, not to care of where the 
function is, which platform the function hoisted, how to learn the interface parameters and how to code a program to quote such function. Such grid resource is simple and standard [1]. However, each desktop software is very different with others.

Desktop processing software package is formed by many functions, which have been integrated and can be use by integration interface or can be use directly with command line mode. The grid transfer of desktop software package is a course of reconstruct, shown in Fig. 1. We think it is no sense to say software package grid modify. Within the grid, a whole application software package cannot be a useful and searchable grid resource to users, because any gird resource should be registered with their resource description according to formal attributions. A large software package has to be separated into function pieces, which can be recognized with their resource registration.

Granularity is real a problem we have to discuss. However, there is less research works has been taken on it. Generally, there are three-type function in software package, processing type, engine type and interface type. Processing type function can realize a certain operation according to arithmetic coding, which always can be run independent. Engine type builds the framework of package and supports common basic function, such as runtime environment, file system and display, while engine type function cannot be use independent. Interface type function contributes to the data format transfer and develops API, some of which can be use as a single function. Within these three types, only processing type and part interface type functions are valuable to grided, which can be mapped to resource tree and managed by grid resource register services.

However, such natural granularity separation is not certain to fit the need of grid service; some special research should be taken for it. But clearly, the fine granularity services can combine into a coarse size granularity service. When considering granularity question, we should pay more attention to the separating difficulty and network performance [2].

The following work is taken with legal PCI develop tools without accessing PCI source code. Such develop tools is also been offered with the purchase of whole package.

\section{Mainframe}

As above said, what can be managed in gird is only the fine-granularity services which separated from PCI package. Such fine-granularity services combine to be a whole middleware layer of PCI, which can be named the gird version of PCI. So, the research work on PCI package grid research can bring a normal method for other desktop remote sensing and GIS processing software package.

In general, PCI hosted workstation can use Windows or UNIX platform. Being a grid node, the server should install basic grid protocols and support services, which bring this node to be a grid node, such as a Globus GT3 environment. But if a PCI hosted workstation is configured as a standard grid node, this grid support services will heavily increase the load, which will affect the normal processing performance of PCI function. So, an independence agent node is needed to be a bridge of grid world and PCI node. Agent node locates in the same LAN with PCI node, they communi- 
cate with a couple of socket program. Agent can be a less capability computer, which installed as a grid node in the same time. As shown in Fig. 2, the resource registered in grid is such agent node instead of the real PCI node. If a grid user wants to use a PCI services, what he quotes is the agent shell services, which will transfer real operation command to the PCI background.

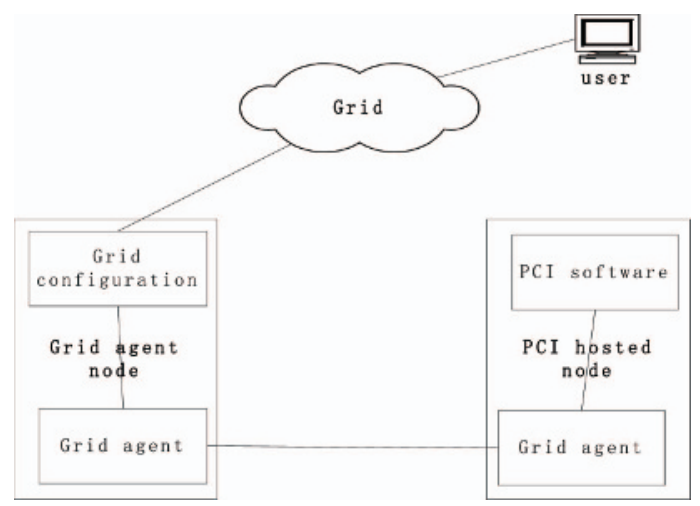

Fig. 2. PCI function grided frame structure

In this structure, PCI node is not changed for the temporary using of grid, such resource can be protecting as independence whole. In fact, it is the real value of grid. Out-grid-sharing will not bring security problem; the upgrade of PCI version also can be hidden to gird user if only keeps the PCI services interface not changed.

The realization of such desktop software function, all can be separated into the following five steps: Enter-parameters formatting (EPF), Enter-image formatting (EIF), quoting PCI function (QF), Outer-image formatting (OIF) and Out-parameters formatting (OPF), shown in Fig. 3. There are two type of information exchanged within grid and desktop software function, operation parameters and image data files' URL. Such information is designed in format of XML. EPF transfers the parameters in XML stream into format of PCI accepted, while EIF will access the source data files (or sub files or database) and replica them to local node via agent node, then EIF also need to change such files into the file format which PCI can handle, such pix file format. OPF and OIF give a reversed operation, which transfer result parameters and files into grid accessible format and return to grid runtime environment. Middle part is $\mathrm{QF}$, which is a standard command line quoting of PCI functions.

\section{Implementation and Experiment}

We designed some experiments about PCI functions in Globus/OGSA environment with application server as JDK. PCI software hosted in a PC workstation configures with Pentium4 $2.0 \mathrm{GHz}$ and $256 \mathrm{MB}$ memory. The agent node is a normal PC computer, which connects PCI node with 100M LAN. Fuzzy classification has been taken as an example with a whole scene TM data, about 40MB, transferred from 
another grid node. Experiment shows that the cost in EPF, EIF, OPF and OIF is $45 \mathrm{sec}$., and QF costs $220 \mathrm{~min}$.

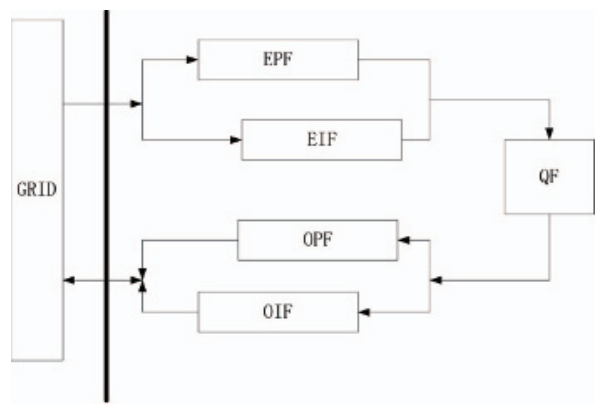

Fig. 3. Five steps of function grided realization

\section{Conclusion}

Above all, desktop spatial software package can be grided modification successfully. Such modification of software package is realized in form of function grided, instead of whole package grided. Grided software can reach resource sharing and operation control. The additional cost is acceptable and processing efficiency is similar between classical method and grid method.

\section{Acknowledgement}

This paper is fund by China National Key Research Project (863) "spatial information grid key technology research and application" and finished at the open laboratory, RSGS, CAS.

\section{References}

1. Bart Jacob Taking advantage of Grid computing for application enablement, IBM technical report. http://www-900.ibm.com/developerWorks/cn/grid/gr-overview/index_eng.shtml

2. Li Guoqing, Research work on spatial information application grid technology, doctoral dissertation, RSGS, CAS, 2004 